\title{
Further Enlargement from Nadir
}

National Cancer Institute

\section{Source}

National Cancer Institute. Further Enlargement from Nadir. NCI Thesaurus. Code C156594.

An increase in size, volume, or quantity in comparison to a previous state that was enlarged from nadir. 